\title{
RM51
}

\section{Parameter Sensitivity in Seismic Net Pay Workflow}

B. Dutton* (Blueback Reservoir)

\section{SUMMARY}

An investigation to illustrate the relative importance of the controlling factors in estimating seismic net pay from colour-inverted band-limited impedance data has been performed. This was achieved by using a new, semi-automated implementation of the process, allowing the sensitivity of each of the key controls on the resulting net pay to be found quickly and easily. This automated workflow was run over a synthetic dataset varying different input parameters. The chi angle and the low frequencies used in building the tuning wedge were found to have the largest effect on the final volume. Using this technique on real datasets could greatly improve the understanding of the possible range of pay volumes, reducing risk and highlighting the key input parameters.

The presentation will work through one manual iteration of the complete workflow, then show how this workflow can be automated. The results running the workflow on a synthetic dataset will be described and discussed. 
The seismic net pay workflow is a method for estimating net pay from seismic data, which takes into account any tuning effects that may alter the apparent reservoir thickness. It comprises of three key building blocks: extended elastic impedance, EEI, (Whitcombe et al. 2002), coloured inversion (Lancaster and Whitcombe 2000) and seismic net pay analysis (Connolly 2007, Simm 2009). The workflow is simple, transparent and robust, and is routinely used in the industry (e.g. Coggins 2011). Running a single pass through the workflow is straightforward and relatively simple to do. However, some steps can be labour intensive, requiring the user to push results from one step to the next, often between applications, making it unrealistic to execute many iterations of the workflow. This makes exhaustive parameter testing impractical.

This presentation utilises a new automated implementation of the workflow ${ }^{1}$ which allows the various input parameters to be investigated by introducing variables and program logic to loop through combinations of inputs. This means many permutations can be tried at the push of a button. It allows questions such as "what ranges of outputs are possible?", "how sensitive is the workflow to parameter $\mathrm{X}$ ?" etc to be answered.

The workflow was run repeatedly on a synthetic dataset, and the total net pay volumes for each iteration recorded. The sensitivity of each parameter on the final predicted net pay volume could be calculated and understood, and the possible ranges of total net pay volumes to be determined. The result is better understanding of the process, reducing the risk and helping better decisions on reservoir development to be made.

The aim is not to find some far-reaching 'rules' about the relative importance of the seismic net pay workflow parameters, rather to show how the sensitivity to the various parameters can be investigated by automatically running the workflow many times. Which parameters are more important will undoubtedly vary from case to case. The ranges of values used in this study were largely arbitrary; with a deeper knowledge of each study area, the ranges of parameters used could be better constrained, thus producing more reliable high/low net pay volumes.

\footnotetext{
${ }^{1}$ Blueback Seismic Reservoir Characterisation Toolbox plugin, running on Petrel*. Available from the Ocean* Store http://www.ocean.slb.com

* Marks of Schlumberger
} 